\title{
Performance Analysis of Cooperative Spectrum Sensing under Guaranteed Throughput Constraints for Cognitive Radio Networks
}

\author{
H. F. Al-Doseri and M. A. Mangoud \\ Department of Electrical and Electronics Engineering, University of Bahrain, P.O. Box 32038, Isa Town, Bahrain \\ Correspondence should be addressed to M. A. Mangoud; mohab@vt.edu
}

Received 7 November 2015; Revised 23 March 2016; Accepted 10 April 2016

Academic Editor: Rui Zhang

Copyright ( 2016 H. F. Al-Doseri and M. A. Mangoud. This is an open access article distributed under the Creative Commons Attribution License, which permits unrestricted use, distribution, and reproduction in any medium, provided the original work is properly cited.

\begin{abstract}
One of the main challenges in cognitive radio networks is the ability of secondary users to detect the primary user presence with high probability of detection. In previous research, optimizing cooperative sensing in cognitive radio networks is performed for either a targeted probability of detection or a false alarm. After setting one of the probabilities as an optimization constraint, the other is optimized. In this paper, a guaranteed constant throughput at the secondary users is introduced as a target while optimizing probability of detection for cooperative sensing. Both sensing time values and number of cooperated cognitive radio secondary users are investigated to maximize the probability of detection of primary user. AND and OR hard decision schemes are considered and compared with soft decision scheme which is weighted modified deflection coefficient scheme (W-MDC). It is illustrated that cooperation of all users and utilizing full frames for sensing time will not provide maximum probability of detection. A tradeoff between performances of cognitive radio networks with and without optimization is presented. The effects of varying network sizes, normalized target throughput, maximum frame duration times, and received signal-to-noise ratio at the fusion center are investigated for different fusion rules.
\end{abstract}

\section{Introduction}

Cognitive radios (CRs) have been widely considered as a promising solution to efficiently utilize the radio spectrum by allowing secondary users (SUs) to access the spectrum of licensed primary users (PUs) [1,2]. Recently, the Federal Communications Commission (FCC) has opened the TV white space (TVWS), which is the unused TV band in time and space for cognitive radios [3]. Consequently IEEE has formed various working task groups (TGs) such as IEEE 802.11af, $802.15 .4 \mathrm{~m}, 802.19 .1$, and $802.22 \mathrm{~b}$ to regulate the unlicensed applications of underutilized TVWS. One of the main challenges of these CRs is that the operation of SUs needs to be maintained at maximum possible throughput without causing disruptive interference to the PUs; this is known as sensing-throughput tradeoff [4]. Cooperative spectrum sensing with different fusion schemes is used to overcome many problems facing the individual sensing to achieve the optimum probability of detection $\left(P_{d}\right)$ and probability of false alarm $\left(P_{f}\right)$. Previously, it is demonstrated that there is an optimal number of cooperated users less than the total network size that gives the best performance of a secondary users network using AND and OR hard decision fusion rules [5]. Further CR network performance enhancement was achieved by applying linear cooperation of local test statistics [6], where modified deflection coefficients (MDC) were optimized to find the weight vector that combats the distractive channel effects. Apparently, soft decision is superior to hard decision since it imposes more sensing information between the CR users and the fusion center (FC). Cooperative spectrum sensing literature includes many research contributions to maximize the channel efficiency or the normalized SUs throughput by optimizing thresholds values, number for SUs, and detection/sensing time [7-11]. 
Earlier optimization studies of optimizing cooperative users' numbers focus on the primary users' perspective and minimize $P_{f}$ under the constraint of fixed $P_{d}$ as constant detection rate (CDR) case in [4]. Other studies lay emphasis on the problem of designing the sensing slot duration and maximize the achievable throughput for the secondary users under the constraint that the primary users are sufficiently protected [7]. However, when targeting fixed probability of detection, this does not guarantee a constant throughput for secondary users. In this paper, we look at the optimization problem from SUs point of view while satisfying the PU requirement. The main contributions of this paper are as follows. A simultaneous optimization of both number of users and sensing time under novel constraint will be presented. The target will be achieving high protection of primary user $\left(P_{d}\right)$ under a guaranteed normalized throughput for secondary users. Furthermore, performances of different CR network scenarios are compared and sensing-throughput tradeoff is discussed for different fusion schemes. Hard decision fusion rules AND and OR are compared with weighted modified deflection coefficient scheme (W-MDC) soft decision rule. Investigations of effect of network sizes, targeted throughput values, total sensing/detection time frames, and received SNR levels are to be presented. The rest of the paper is organized as follows. Section 2 presents network model description and normalized SUs throughput definition. In Section 3, expressions for $P_{d}$ are derived under constant normalized throughput constraint for different fusion schemes to formulate the optimization problem. Optimizing numbers of cooperative users and sensing time for CRs will be discussed in Section 4 along with investigations of the effect of key parameters such as network size, targeted efficiency rate, total sensing time, and received SNR levels. Finally, the paper will be concluded in Section 5 .

\section{Network Model and Throughput Tradeoff}

2.1. Single User Energy Detection. Suppose a cognitive radio network with $M$ cooperative secondary users and one fusion center that make a decision on the primary user activity based on the received decisions from the different secondary users. Assume that energy detection is utilized at each single secondary user (SU). Let $H_{o}: x_{i}(n)=u(n)$ and $H_{1}: x_{i}(n)=$ $s(n)+u(n)$ be the hypotheses of the primary user being inactive or active, respectively. $x_{i}(n)$ is the received signal by the $i$ th SU at sample $n . s(n)$ is the PU signal and it is assumed to be independent and identically distributed (i.i.d.) random process with zero mean and variance of $\sigma_{s}^{2} . s(n)$ is assumed to be a complex PSK modulated signal.

$u(n)$ is the noise signal that is considered as i.i.d. Gaussian distribution with zero mean and variance of $\sigma_{u}^{2}$.

For a single CR spectrum sensing scheme [5-8], the local decision rule is modelled as

$$
U_{i} \underset{H_{0}}{\stackrel{H_{1}}{\lessgtr}} \lambda_{i} \quad i=1,2, \ldots, M,
$$

where $U_{i}$ is the test statistic of the $i$ th secondary user using energy detection over a detection interval of $N$ samples

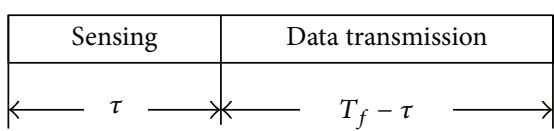

Figure 1: Frame structure of the cognitive radio user.

which is calculated as $U_{i}=(1 / N) \sum_{n=1}^{N}\left|x_{i}(n)\right|^{2}$ and $\lambda_{i}$ is the corresponding decision threshold, since $U_{i}$ is the sum of squares of $N$ Gaussian random variables. It is shown previously in [6] that the standard Gaussian variable $U_{i} / \sigma_{u}^{2}$ follows a central chi square $\chi_{N}^{2}$ distribution with $N$ degrees of freedom if $H_{o}$ is true. In case $H_{1}$ is true the standard Gaussian variable $U_{i} / \sigma_{u}^{2}$ would follow a noncentral chi square distribution $\chi_{N}^{2}$ with $N$ degrees of freedom. According to the central limit theorem, if the number of samples $N$ is large enough, test statistics $U_{i}$ are asymptotically Gaussian distributed. Define the SNR at the $i$ th user which is the primary user signal power to noise ratio measured at the SU receiver of interest as $\gamma_{i}=\sigma_{s}^{2} / \sigma_{u}^{2}$. The probabilities of detection and false alarm $\left(P_{d, i}\right.$ and $\left.P_{f, i}\right)$ at the $i$ th secondary user in terms of targeted $\overline{P_{f, i}}$, and $\overline{P_{d, i}}$ and sampling size $N$, as introduced in [3], are given by

$$
\begin{aligned}
P_{d, i} & =\operatorname{Pr}\left(U_{i}>\lambda_{i} \mid H_{1}\right) \\
& =Q\left(\frac{1}{\sqrt{2 \gamma_{i}+1}} \cdot\left[Q^{-1}\left(\overline{P_{f, i}}\right)+\gamma_{i} \sqrt{N}\right]\right), \\
P_{f, i} & =\operatorname{Pr}\left(U_{i}>\lambda_{i} \mid H_{0}\right) \\
& =Q\left(\sqrt{2 \gamma_{i}+1} \cdot Q^{-1}\left(\overline{P_{d, i}}\right)+\gamma_{i} \sqrt{N}\right)
\end{aligned}
$$

where $Q(\cdot)$ denotes the complementary cumulative distribution function of a zero mean, unit variance Gaussian distribution.

2.2. Throughput Calculations. Cognitive user is considered to operate in a frame basis transmission. As shown in Figure 1, the cognitive user performs $N$ samples in a periodic sensing and transmission frame duration every $T_{f}$; if $T_{s}$ is the sampling time, then $T_{f}=N \cdot T_{s}$. The frame comprises sensing interval time $\tau$ that has $n$ detection samples used for sensing PU. The second part of the frame is the data transmission interval (active or idle) with duration of $\left(T_{f}-\tau\right)$ that has $N-n$ samples, where $1 \leq n \leq N$.

The average normalized achieved throughput of the SU, $R(\tau)$, was previously defined in [8-10] as

$$
\begin{aligned}
& R(\tau)=\frac{T_{f}-\tau}{T_{f}}\left\{\left(1-P_{f}\right) \cdot P\left(H_{o}\right) \cdot \log _{2}\left(1+\mathrm{SNR}_{\mathrm{SU}}\right)\right. \\
& \left.+\left(1-P_{d}\right) \cdot P\left(H_{1}\right) \cdot \log _{2}\left(1+\frac{\mathrm{SNR}_{\mathrm{SU}}}{\mathrm{SNR}_{\mathrm{PU}}}\right)\right\},
\end{aligned}
$$

where $\mathrm{SNR}_{\mathrm{SU}}$ and $\mathrm{SNR}_{\mathrm{PU}}$ are the received signal-to-noise of SU and PU, respectively, at the SU receiver. The first term in (4) is related to the throughput of the SU when it operates (no 
false alarm is generated) in the absence of PU. The second term in (4) considers the scenario when the SU operates in the presence of PU while failing to detect it (no detection). It is usually assumed that if the primary users require $100 \%$ protection in its frequency band, it will then be not allowed for the secondary usage in that frequency band. Hence, it is assumed that the first term in the right hand side dominates the achievable throughput.

Therefore the normalized achievable throughput of the ith SU can be approximated by

$$
R_{n}(\tau)=\frac{T_{f}-\tau}{T_{f}}\left(1-P_{f, i}\right) .
$$

As can be seen, the average normalized throughput of the energy detector depends on the ratio of the amount of time that the SU channel can be used for transmission to the total frame time. The normalized throughput $R_{n}(\tau)$ is equivalent to the channel efficiency of the SU that was defined in [11] as

$$
\eta(n)=\frac{N-n}{N}\left(1-P_{f, i}\right),
$$

where the number of samples $(n$ and $N)$ is used here instead of time parameters $\left(\tau\right.$ and $T_{f}$ ). The average achieved normalized throughput in terms of $i$ th $\mathrm{SU}, P_{d, i}$ will be expressed by substituting $P_{f, i}$ of (3) into (5) to be

$$
\begin{aligned}
& R_{n}(\tau) \\
& \quad=\frac{T_{f}-\tau}{T_{f}}\left(1-\left(Q\left(\sqrt{2 \gamma_{i}+1} Q^{-1}\left(\overline{P_{d, i}}\right)+\gamma_{i} \sqrt{n}\right)\right)\right),
\end{aligned}
$$

where $n=\tau$. Fs and Fs $=1 / T_{s}$ is the sampling frequency. As can be seen the achievable throughput of the secondary network is a function of the sensing time $\tau$. By intuition using less time for detection enables SU to obtain more time for transmission in a single cycle. However, this may lead to degradation in detection performance. Therefore, tradeoff optimization is needed for sensing time.

\section{Cooperative Spectrum Sensing under Constant Achievable Throughput Condition}

Cooperative spectrum sensing is classified as hard decision or soft decision schemes according to the way the presence of PU is determined at the fusion center with the composed reported decisions from the individual selected SUs. Every SU will make its own decision and transmits one-bit decision to FC that makes a final cooperative decision by fusing the data collected from SUs. For hard decisions, AND and OR schemes are considered in this paper. For soft decision schemes, we choose weighted modified deflection coefficient (W-MDC) [6] in which the FC linearly combines the received statistics and obtains a global test statistic.

3.1. Constant Efficiency Rate Cooperative Spectrum Sensing. In this paper, we introduce the performance analysis of the cognitive network performance under a constant normalized throughput (CNT) condition. Optimization of the sensing duration $(\tau)$ and the required number of cooperative users $(k)$ is performed to maximize $P_{d}$ of the network of size $(M)$. This requirement leads to achieving higher $P_{d}$ that will ensure PU protection and also guaranteeing high constant throughput $\left(\overline{R_{n}}\right)$ for SUs. Targeting a constant efficiency, $P_{f}\left(\overline{R_{n}}, \tau\right)$ can be obtained by reforming (5) into

$$
P_{f}\left(\overline{R_{n}}, \tau\right)=1-\frac{T_{f} \cdot \overline{R_{n}}}{T_{f}-\tau} .
$$

Since we have two constraints, $0 \leq P_{f} \leq 1$ and $0 \leq R_{n} \leq 1$, these constraints will create a new higher bound of $(\tau)$ which is $\tau_{\max }$, the highest allowable window length to achieve the targeted normalized throughput $\overline{R_{n}}$, and it can be calculated by

$$
\tau_{\max }\left(\overline{R_{n}}\right)=T_{f}\left(1-\overline{R_{n}}\right)
$$

3.2. AND Fusion Rule under CNT Requirement. AND decision rule states that the $\mathrm{PU}$ is present when all the selected SUs at FC detect the PU. The total probability of detection and false alarm of the network using decision of $k$ cooperating users are given by

$$
\begin{aligned}
& P_{d, \mathrm{AND}}=\prod_{i=1}^{k} P_{d, i}, \\
& P_{f, \mathrm{AND}}=\prod_{i=1}^{k} P_{f, i},
\end{aligned}
$$

where $P_{d, i}$ and $P_{f, i}$ are the probabilities of detection and false alarm for each individual SU. It should be noted that $k$ is bounded as $1 \leq k \leq M$ since the network size is $M$. To analyze/optimize the performance of AND fusion rule under CNT, an expression of network probability $P_{d, \mathrm{AND}}$ needs to be derived as a function of the targeted normalized throughput $\left(\overline{R_{n}}\right)$, sensing time $(\tau)$, and the number of cooperating users $(k)$. From (11), the individual false alarm rate for the $i$ th SU will be $P_{f, i}=\sqrt[k]{P_{f, \mathrm{AND}}}$. Therefore, the individual detection rate can be obtained from (2) by replacing $\overline{P_{f, i}}$ with the $i$ th SU false alarm from AND fusion rule as

$$
\begin{aligned}
& P_{d, i}(k, \tau) \\
& \quad=Q\left(\frac{1}{\sqrt{2 \gamma_{i}+1}} \cdot\left[Q^{-1}\left(\sqrt[k]{P_{f, \mathrm{AND}}}\right)+\gamma_{i} \sqrt{\tau \cdot \mathrm{Fs}}\right]\right) .
\end{aligned}
$$

By substituting (12) into (10), the probability of detection for the network $P_{f, \mathrm{AND}}$ is

$$
\begin{aligned}
& P_{d, \mathrm{AND}}(k, \tau) \\
& =\prod_{i=1}^{k} Q\left(\frac{1}{\sqrt{2 \gamma_{i}+1}} \cdot\left[Q^{-1}\left(\sqrt[k]{P_{f, \mathrm{AND}}}\right)+\gamma_{i} \sqrt{\tau \cdot \mathrm{Fs}}\right]\right) .
\end{aligned}
$$

With CNT requirement, by fixing the throughput of the networks targeting a constant throughput $\left(\overline{R_{n}}\right)$, (5) can be 
reformed to obtain the network cooperative users false alarm probability as

$$
P_{f, \mathrm{AND}}\left(\overline{R_{n}}, \tau\right)=\left(1-\frac{T \overline{R_{n}}}{T-\tau}\right),
$$

where the sensing time range is $1 \leq \tau \leq \tau_{\max }$ and the number of cooperative users is bounded by $1 \leq k \leq M$; then $1 \leq i \leq k$. Therefore, the individual $P_{f, i}$ for AND fusion scheme in terms of $\left(\overline{R_{n}}, k, \tau\right)$ is

$$
P_{f, i}\left(\overline{R_{n}}, k, \tau\right)=\sqrt[k]{P_{f, \mathrm{AND}}\left(\overline{R_{n}}, \tau\right)}=\sqrt[k]{\left(1-\frac{T \overline{R_{n}}}{T-\tau}\right)} .
$$

$P_{d, i, \mathrm{AND}}$ for the individual $i$ th $\mathrm{SU}$ within an AND cooperation of $(k)$ SUs can be calculated by

$$
\begin{aligned}
& P_{d, i, \mathrm{AND}}\left(\overline{R_{n}}, k, \tau\right) \\
& =Q\left(Q^{-1}\left(\sqrt[k]{\left(1-\frac{T \overline{R_{n}}}{T-\tau}\right)}\right)-\frac{\gamma_{i} \tau \mathrm{Fs}}{\sqrt{2 \gamma_{i}+1}}\right) .
\end{aligned}
$$

The probability of detection for the AND cooperation of $(k)$ users with optimized sensing time and under CNT will be

$$
\begin{aligned}
& P_{d, \mathrm{AND}}\left(\overline{R_{n}}, k, \tau\right) \\
& \quad=\prod_{i=1}^{k} Q\left(Q^{-1}\left(\sqrt[k]{\left(1-\frac{T \overline{R_{n}}}{T-\tau}\right)}\right)-\frac{\gamma_{i} \sqrt{\mathrm{Fs} \cdot \tau}}{\sqrt{2 \gamma_{i}+1}}\right) .
\end{aligned}
$$

Our objective is to optimize this detection rate to guarantee the target throughput $\overline{R_{n}}$ and obtain optimal values of number of cooperative users $(k)$ and sensing window length $(n)$.

3.3. OR Fusion Scheme under CNT Requirement. OR decision rule states that when at least one of the network users detects the primary signal, the FC will state that the $\mathrm{PU}$ is present. $P_{d}$ and $P_{f}$ for OR network can be written as

$$
\begin{aligned}
& P_{d, \mathrm{OR}}=1-\prod_{i=1}^{k}\left(1-P_{d, i}\right), \\
& P_{f, \mathrm{OR}}=1-\prod_{i=1}^{k}\left(1-P_{f, i}\right) .
\end{aligned}
$$

For a probability of false alarm for the network $P_{f, \mathrm{OR}}$, the individual false alarm rate is approximately given by

$$
P_{f, i}=1-\sqrt[k]{\left(1-P_{f, \mathrm{OR}}\right)} .
$$

When OR fusion rule is used, $P_{d}$ of each SU in a cooperation of $k$ secondary users with sensing time window duration $\tau$ can be calculated by

$$
\begin{gathered}
P_{d, i}(k, \tau)=Q\left(\frac{1}{\sqrt{2 \gamma_{i}+1}}\right. \\
\left.\cdot\left[Q^{-1}\left(1-\sqrt[k]{\left(1-P_{f, \mathrm{OR}}\right)}\right)+\gamma_{i} \sqrt{\tau \cdot \mathrm{Fs}}\right]\right) .
\end{gathered}
$$

The network $P_{d, \mathrm{OR}}$ is then calculated by substituting (21) into (18):

$$
\begin{gathered}
P_{d, \mathrm{OR}}(k, \tau)=1-\prod_{i=1}^{k}\left(1-\mathrm{Q}\left(\frac{1}{\sqrt{2 \gamma_{i}+1}}\right.\right. \\
\left.\left.\cdot\left[\mathrm{Q}^{-1}\left(1-\sqrt[k]{\left(1-P_{f, \mathrm{OR}}\right)}\right)+\gamma_{i} \sqrt{\tau \cdot \mathrm{Fs}}\right]\right)\right),
\end{gathered}
$$

where $1 \leq \tau \leq \tau_{\max }$ and $1 \leq k \leq M$. If a guaranteed achievable normalized throughput, $\overline{R_{n}}$, is considered (i.e., CNT condition), form (5) and (20), the probabilities of false alarm of the OR network and the individual ith SU are

$$
\begin{aligned}
P_{f, \mathrm{OR}}\left(\overline{R_{n}}, k, \tau\right) & =1-\left(1-P_{f, i}\right)^{k}=1-\frac{\overline{R_{n}} \cdot T_{f}}{T_{f}-\tau}, \\
P_{f, i}\left(\overline{R_{n}}, k, \tau\right) & =1-\sqrt[k]{\left(1-P_{f, \mathrm{OR}}\left(\overline{R_{n}}, \tau\right)\right)} \\
& =1-\sqrt[k]{\frac{\overline{R_{n}} \cdot T_{f}}{T_{f}-\tau}} .
\end{aligned}
$$

$P_{d}$ for the $i$ th $\mathrm{SU}$ in a cooperation of $(k)$ SUs at a frame length $(n)$ using OR fusion rule can be written as

$$
\begin{aligned}
& P_{d, i}\left(\overline{R_{n}}, k, \tau\right) \\
& \quad=Q\left(Q^{-1}\left(1-\sqrt[k]{\frac{\overline{R_{n}} \cdot T_{f}}{T_{f}-\tau}}\right)-\frac{\gamma_{i} \sqrt{\tau \cdot \mathrm{Fs}}}{\sqrt{2 \gamma_{i}+1}}\right) .
\end{aligned}
$$

And $\left(P_{d}\right)$ for the OR cooperation of $k$ users is

$$
\begin{aligned}
P_{d, \mathrm{OR}} & \left(\overline{R_{n}}, k, \tau\right) \\
= & 1 \\
& \quad-\prod_{i=1}^{k} \mathrm{Q}\left(\mathrm{Q}^{-1}\left(1-\sqrt[k]{\frac{\overline{R_{n}} \cdot T_{f}}{T_{f}-\tau}}\right)-\frac{\gamma_{i} \sqrt{\tau \cdot \mathrm{Fs}}}{\sqrt{2 \gamma_{i}+1}}\right)
\end{aligned}
$$

which is our target close form for the network detection rate for OR fusion scheme in terms of $\overline{R_{n}}, k, \tau$ under CNT that is needed to provide network performance and will be used in Section 4 .

3.4. Weighted Modified Deflection Coefficients Scheme under CNT Requirement. Another decision rule category is the soft decision type. An example is weighted MDC (W-MDC) scheme. In [6], a linear cooperation for spectrum sensing that optimizes modified deflection coefficient (MDC) was proposed to find the weight vector for all possible cognitive radios. Significant cooperative gain was achieved compared to hard decision schemes. In this paper, performance of 


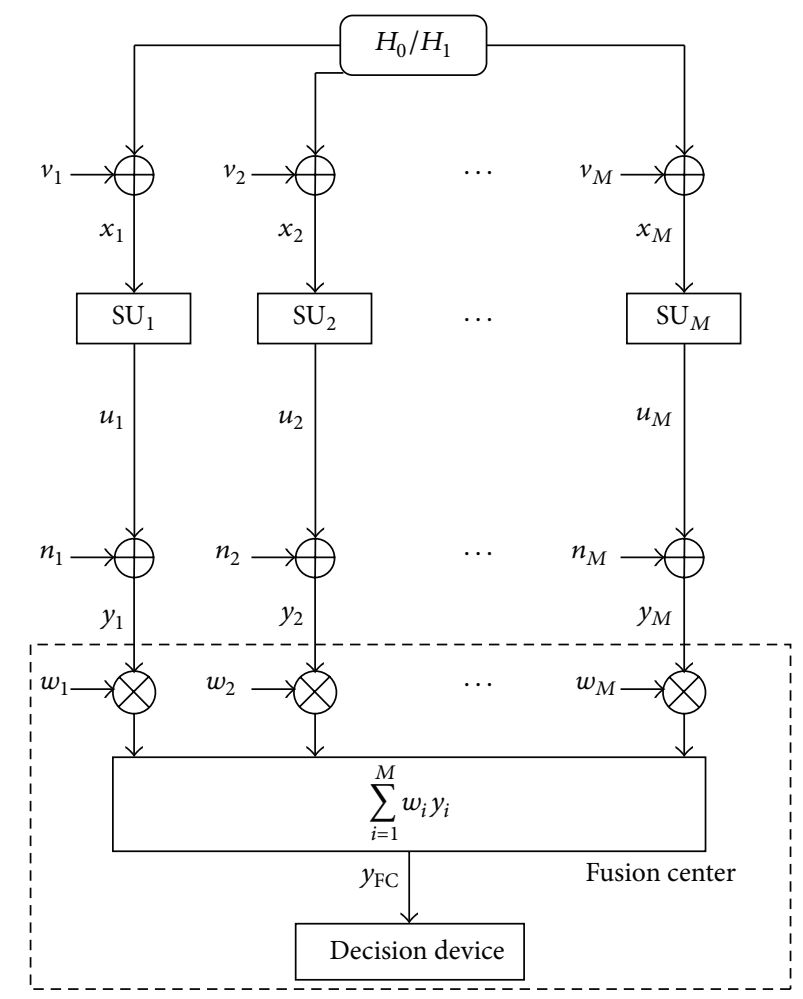

Figure 2: System model for weighted cooperative spectrum sensing.

W-MDC will be considered under CNT requirement, by targeting a fixed normalized throughput for the cognitive network, $\overline{R_{n}}$.

The system model of cognitive network with W-MDC scheme is shown in Figure 2. The network is assumed to be composed of $(M)$ SUs and the binary hypotheses for spectrum sensing at the $n$th sample are

$$
\begin{aligned}
& H_{0}: x_{i}(n)=v_{i}(n), \\
& H_{1}: x_{i}(n)=h_{i} s(n)+v_{i}(n),
\end{aligned}
$$

where $x_{i}$ is the received signal by the $i$ th $\mathrm{SU}, s$ is the signal transmitted by the PU, $h_{i}$ is the channel gain between the PU signal source and the $i$ th SU which is assumed to be constant at the detection interval, and $v_{i}$ is the sensing channel noise for the $i$ th $\mathrm{SU}$ which is assumed to be zero-mean additive white Gaussian noise (AWGN) with variances $\sigma_{i}^{2}$ and all variances are collected into a vector $\boldsymbol{\sigma}=\left[\sigma_{1}^{2}, \sigma_{2}^{2}, \ldots, \sigma_{M}^{2}\right]$. At each SU using energy detector, the summary statistic $u_{i}$ is generated by summation of the received signal energy over the detection interval $N_{\text {det }}$ as follows:

$$
u_{i}=\sum_{n=1}^{N_{\mathrm{det}}}\left|x_{i}(n)\right|^{2}
$$

for $i=1,2, \ldots, M$. The summary statistics from all users are transmitted to the FC through the reporting channel; the received signals at the FC are represented by

$$
\left[\begin{array}{c}
y_{1} \\
y_{2} \\
\vdots \\
y_{M}
\end{array}\right]=\left[\begin{array}{c}
u_{1} \\
u_{2} \\
\vdots \\
u_{M}
\end{array}\right]+\left[\begin{array}{c}
n_{1} \\
n_{2} \\
\vdots \\
n_{M}
\end{array}\right] \text {, }
$$

where $n_{i}$ is the reporting channel noise for the $i$ th SU which is assumed to be a zero-mean AWGN with variance $\delta_{i}^{2}$. The variances of the reporting channels are collected into a vector $\boldsymbol{\delta}=\left[\delta_{1}^{2}, \delta_{2}^{2}, \ldots, \delta_{M}^{2}\right]$. After adding weights to the received signals at the FC, the final test statistic is

$$
y_{\mathrm{W}-\mathrm{MDC}}(k)=\sum_{i=1}^{k} w_{i} y_{i}=\mathbf{w}^{T} \mathbf{y}
$$

where $\mathbf{w}=\left[w_{1}^{2}, w_{2}^{2}, \ldots, w_{k}^{2}\right]^{T}$ and $\mathbf{y}=\left[y_{1}, y_{2}, \ldots, y_{k}\right]$, where $T$ denotes the transpose of the vector, $w_{i} \geq 0$. From the work of [6], the means of the test statistic at the FC under different hypotheses are

$$
\bar{y}_{\mathrm{W}-\mathrm{MDC}}= \begin{cases}T_{f} \cdot \mathrm{Fs} \cdot \boldsymbol{\sigma}^{T} \mathbf{w}, & H_{0} \\ \left(T_{f} \cdot \mathrm{Fs} \cdot \boldsymbol{\sigma}+E_{s} \mathbf{h}\right)^{T} w, & H_{1},\end{cases}
$$


where $E_{s}=\gamma_{i} \sigma_{i}^{2} /\left|\mathbf{h}_{i}\right|^{2}, \mathbf{h}=\left\{\left|h_{1}\right|^{2}, \ldots,\left|h_{k}\right|^{2}\right\}^{T}$. Defining $\mathbf{A}=$ $2 N \operatorname{diag}^{2}(\boldsymbol{\sigma})+\operatorname{diag}(\boldsymbol{\delta})$ and $\mathbf{B}=\mathbf{A}+4 E_{s} \operatorname{diag}(\boldsymbol{\sigma}) \operatorname{diag}(\boldsymbol{\delta})$, the variances are

$$
\sigma_{\mathrm{W}-\mathrm{MDC}}^{2}= \begin{cases}\mathbf{w}^{T} \mathbf{A w}, & H_{0} \\ \mathbf{w}^{T} \mathbf{B w}, & H_{1} .\end{cases}
$$

$\left(P_{d}\right)$ of the weighted SU network can be represented as a function of $\left(P_{f}\right)$ as

$$
P_{d}=Q\left(\frac{Q^{-1}\left(P_{f}\right) \sqrt{\mathbf{w}^{T} \mathbf{A} \mathbf{w}}-E_{s} \mathbf{h}^{T} \mathbf{w}}{\sqrt{\mathbf{w}^{T} \mathbf{B w}}}\right) .
$$

Since the weight vector is an important parameter affecting the $\left(P_{d}\right)$, a modified deflection coefficient (MDC) was presented as

$$
\begin{aligned}
d_{m}^{2}(w) & =\frac{\left[E\left(y_{\mathrm{W}-\mathrm{MDC}} \mid H_{1}\right)-E\left(y_{\mathrm{W}-\mathrm{MDC}} \mid H_{0}\right)\right]^{2}}{\operatorname{Var}\left(y_{\mathrm{W}-\mathrm{MDC}} \mid H_{1}\right)} \\
& =\frac{\left(E_{s} \mathbf{h}^{T} \mathbf{w}\right)^{2}}{\mathbf{w}^{T} \mathbf{B} \mathbf{w}} .
\end{aligned}
$$

The optimization problem of the MDC is $\left\{\max _{\mathbf{w}} d_{m}^{2}(\mathbf{w})\right\}$ so that $\|\mathbf{w}\|_{2}^{2}=1$, which will lead to the optimal solution:

$$
\mathbf{w}_{\mathrm{opt}}=\frac{\mathbf{B}^{-1 / 2} \mathbf{B}^{-T / 2} \mathbf{h}}{\left\|\mathbf{B}^{-1 / 2} \mathbf{B}^{-T / 2} \mathbf{h}\right\|_{2}} .
$$

The network detection performance of W-MDC under CNT is obtained by substituting (8) in (32). Therefore, the detection rate as a function of $\overline{R_{n}}, k$, and $\tau$ will be given by

$$
\begin{aligned}
& P_{d, \mathrm{~W}-\mathrm{MDC}}\left(\overline{R_{n}}, k, \tau\right) \\
& =\mathrm{Q}\left(\frac{\mathrm{Q}^{-1}\left(1-\left(\left(\overline{R_{n}} \cdot T_{f}\right) /\left(T_{f}-\tau\right)\right)\right) \sqrt{\mathbf{w}^{T} \mathbf{A w}}-E_{s} \mathbf{h}^{T} \mathbf{w}}{\sqrt{\mathbf{w}^{T} \mathbf{B w}}}\right) .
\end{aligned}
$$

\section{Numerical Results and Analysis}

In this section, computer simulation results are presented to evaluate the overhead throughput tradeoff by finding the optimal sensing time and the optimal number of secondary users. This is performed to maximize the secondary users network performances under constant achievable normalized throughput constraint. Different fusion rules, network sizes $(M)$, targeted throughput $(R)$, frame duration $(T)$, and received signal-to-noise ratio levels (SNR) are considered. The cognitive radio network model considered in the simulations is illustrated in Figure 3, where the received SNR at the location of the fusion center (FC) is $-14 \mathrm{~dB}$; the primary user (PU) is $120 \mathrm{~km}$ away from the fusion center (FC) which is considered to be placed at the center of the secondary users' (SUs) network. The SU network is assumed to be randomly distributed in a $900 \mathrm{~km}^{2}$ square in the vicinity of the FC, the path loss exponent is set to 3.2, the total window length of the full frame of the $\mathrm{SU}$ is assumed to

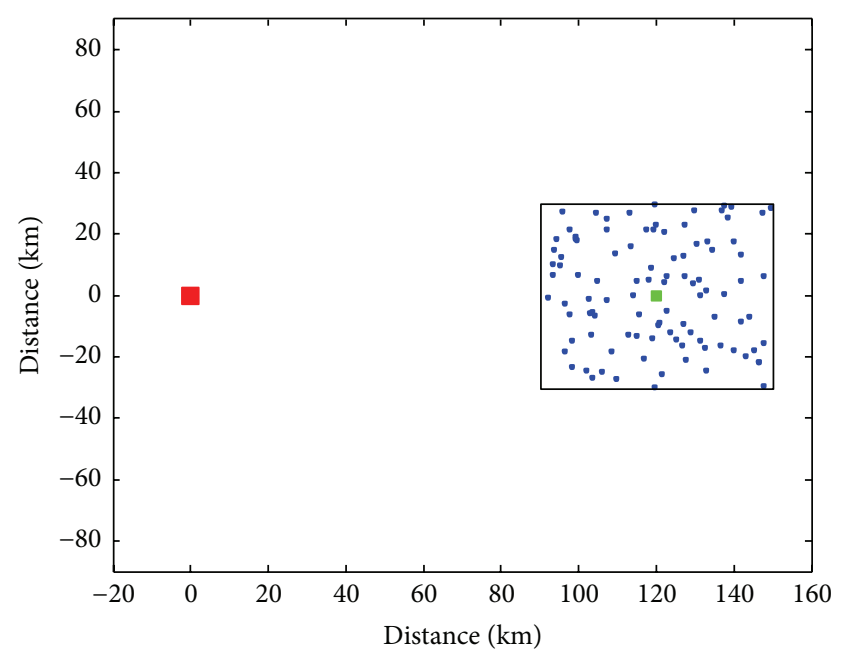

- Primary user

- Fusion center

- Secondary user

FIGURE 3: Simulation scenario for cooperative cognitive network with 100 secondary users, primary user, and fusion center.

be 1000 samples with sampling frequency $\left(f_{s}=1 \mathrm{MHz}, T_{s}\right.$ $=1 \mu \mathrm{s})$, and total time frame $(T)$ is $1 \mathrm{~ms}$. The following results are obtained by averaging each simulation run over 20 random network distributions. Simulations are carried out to optimize the network performance under constant normalized throughput (CNT) constraint as described in Section 3. The normalized targeted throughput is assumed to be 0.9 . In this case, $P_{f}=(1-(T R /(T-\tau)))$. Since we have two constraints: $0 \leq P_{f} \leq 1$ and $0 \leq R \leq 1$, a new higher bound of sensing time will be considered which is the highest allowable sensing time $\left(\tau_{\max }\right)$ to achieve the targeted normalized throughput and it can be calculated as $\tau_{\max }=T(1-R)$. Since $T$ is assumed to be $1 \mathrm{~ms}$ and $R=0.9$, $\tau_{\max }=100 \mu \mathrm{s}$.

Figures 4(a), 4(b), and 4(c) show the performances of the SU network with AND, OR, and W-MDC weighted rules, respectively, with network size $(M)$ equal to 100. As can be seen for every case, there exist an optimal number of cooperative users $\left(k_{\text {opt }}\right)$ which is less than the $(M)$ and an optimal sensing time $\left(\tau_{\text {opt }}\right)$ which is less than the maximum allowable detection time $\left(\tau_{\max }\right)$ under constant normalized throughput constraint to achieve the maximum performance. The contours behaviors show the improvement of CNT-OR network performance when the number of secondary users having relatively high SNR levels is increasing. The contour lines are used to show the optimal values of $\left(k_{\mathrm{opt}}, \tau_{\mathrm{opt}}\right)$ pairs to achieve the required throughput. It is noticed that, with the optimum values of $\left(k_{\text {opt }}, \tau_{\text {opt }}\right)$, the maximum $\left(P_{d}\right)$ achieved by optimizing OR network is higher than that achieved by AND network. MDC weighting sensing outperforms both $\mathrm{OR}$ and AND as it achieves higher $P_{d}$ values; however, the cooperation of all users $\left(k_{\mathrm{opt}}=M\right)$ is needed to achieve the maximum performance.

The performance of the SU network with different network sizes $(M)$ under constant normalized throughput requirements is investigated and illustrated in Figure 5. 


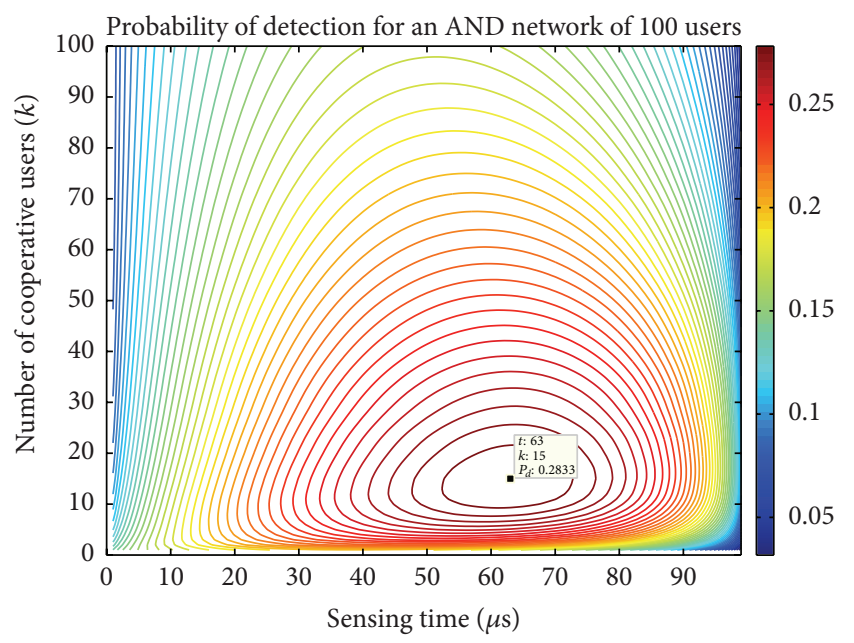

(a)

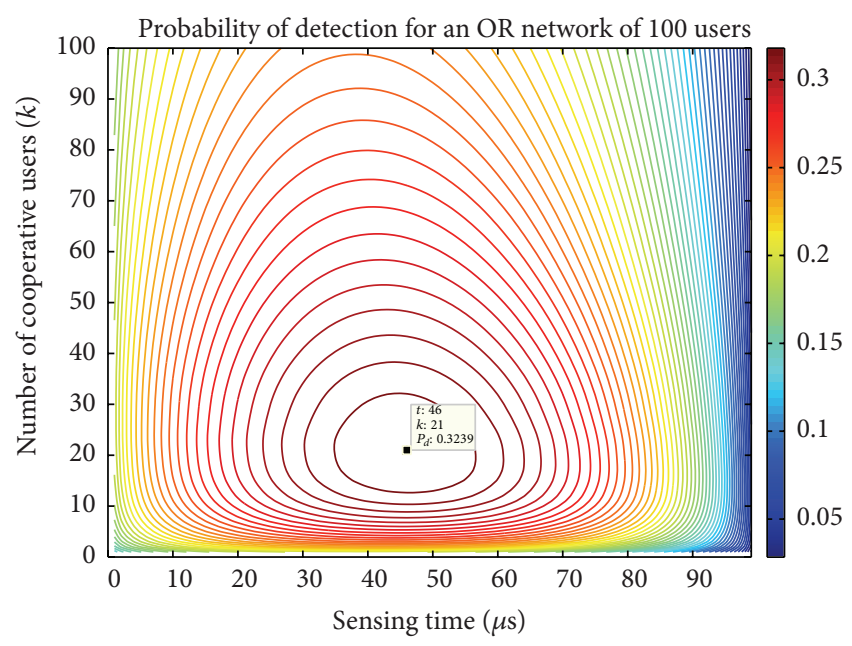

(b)

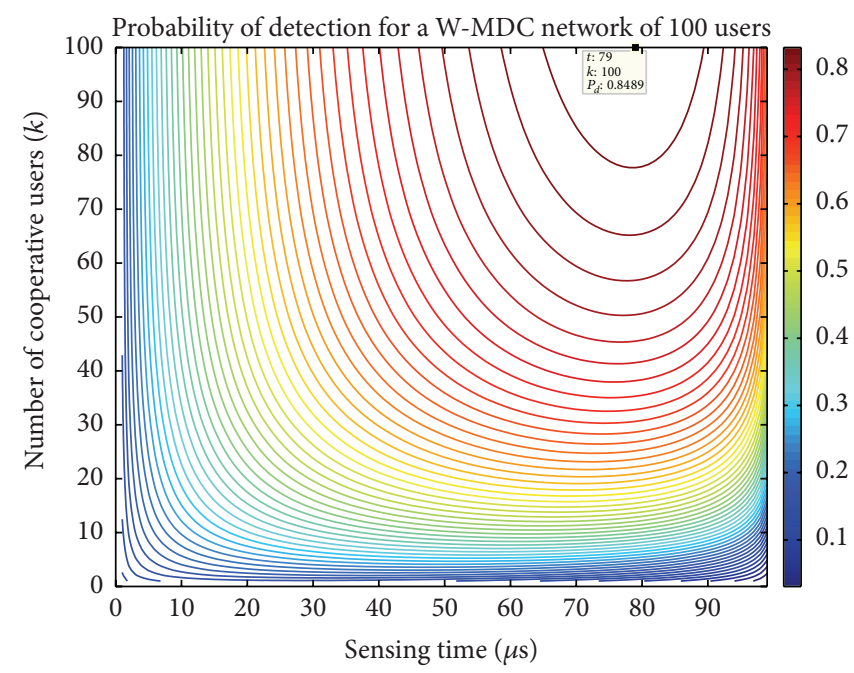

(c)

FIGURE 4: Probability of detection $\left(P_{d}\right)$ versus sensing time and number of cooperative users for (a) AND, (b) OR, and (c) weighted MDC fusion scheme under CNT condition of $R=0.9$ with $M=100$ and $\tau_{\max }=100 \mu \mathrm{s}$.

Network size effect on the performance has been investigated with the number of secondary users set to be between 25 and 200 for target normalized throughput of 0.9. The figure shows $P_{d}$ of the network with optimized number of users and sensing times $\left(k_{\mathrm{opt}}, \tau_{\mathrm{opt}}\right)$ for different fusion schemes under CNT requirement. Results are compared with the cases where all SUs are cooperated and maximum sensing time is utilized. Results show that optimizing $k$ and $\tau$ will lead to better detection performance than cooperating all the SUs in the network. As can be seen, higher probabilities of detection $\left(P_{d}\right)$ are achieved as the network size $(M)$ increases. The WMDC achieves higher values of $P_{d}$ compared to AND and OR schemes. However, W-MDC requires longer sensing time and more cooperative SUs to achieve its optimal performance than AND and OR. For a network consisting of 100 SUs the best $P_{d}$ is obtained with $\left(k_{\mathrm{opt}}, \tau_{\mathrm{opt}}\right)=(15,63),(21,46)$, and $(100,79)$ for AND, OR, and W-MDC, respectively. It is also concluded that optimizing both $k$ and $\tau$ leads to improving $P_{d}$ while maintaining the desirable fixed throughput.

Figure 6 compares the optimum $P_{d}$ with the unoptimized conventional $P_{d}$ when all the secondary users are used for cooperation at different normalized throughput $(R)$ in the range from 0.85 to 0.95 . When AND, OR, and W-MDC fusion schemes are used in a network consisting of 100 secondary users, previous simulation SUs network parameters such as distribution area, path loss exponent, total sensing time, and SNR at FC are kept unchanged. Also, the optimum values of $\left(k_{\mathrm{opt}}, \tau_{\mathrm{opt}}\right)$ are presented for each case. As shown in the figure, as the targeted normalised throughput $(R)$ increases, degraded network performance is observed.

The effect of changing the total time frame duration $(T)$ on the primary user detection performance for the network of 100 SUs with the same simulation parameters is demonstrated in Figure 7. In general, increasing total time frame duration $(T)$ will enhance the network performance. Also, it is shown 


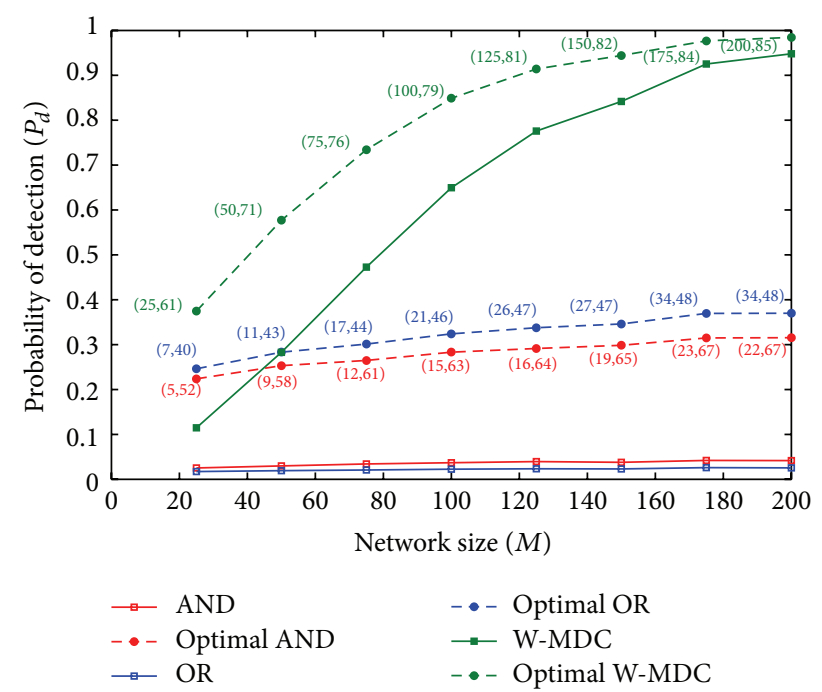

FIGURE 5: The probability of detection $\left(P_{d}\right)$ versus network size $(M)$ for AND, OR, and weighted MDC fusion scheme; network and targeted normalized throughput $=0.9$ and $\tau_{\max }=100 \mu$ s with $\left(k_{\mathrm{opt}}, \tau_{\mathrm{opt}}\right)$ values are shown in the data tips.

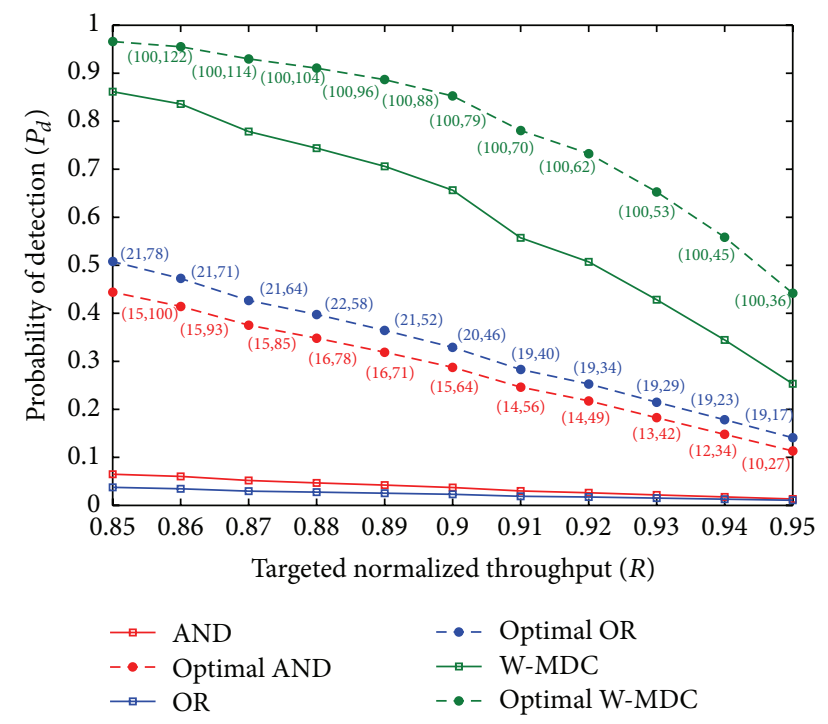

FIGURE 6: The probability of detection $\left(P_{d}\right)$ versus targeted normalized throughput for AND, OR, and weighted MDC fusion scheme; network with 100 SUs and maximum sensing time of $\tau_{\max }=100 \mu \mathrm{s}$ with $\left(k_{\text {opt }}, \tau_{\text {opt }}\right)$ are shown in the data tips.

that, for a given frame duration, optimizing number of cooperated users $(k)$ and sensing time $(\tau)$ will increase the system performance, for example, nonoptimized case with $T=1000 \mu \mathrm{s}, P_{d}<0.05$, for AND and OR and $P_{d}=0.62$ for W-MDC case. However, when optimizing the sensing time and number of cooperative users for the same frame duration, $P_{d}$ becomes $\approx 0.3$ which has been achieved for AND and OR schemes, and $P_{d}$ is increased to 0.82 for W-MDC with optimum values of $k_{\text {opt }}=14,20$, and 100 cooperated users and $\tau_{\mathrm{opt}}=62,45$, and $78 \mu$ s for AND, OR, and WMDC schemes respectively, respectively. When increasing the

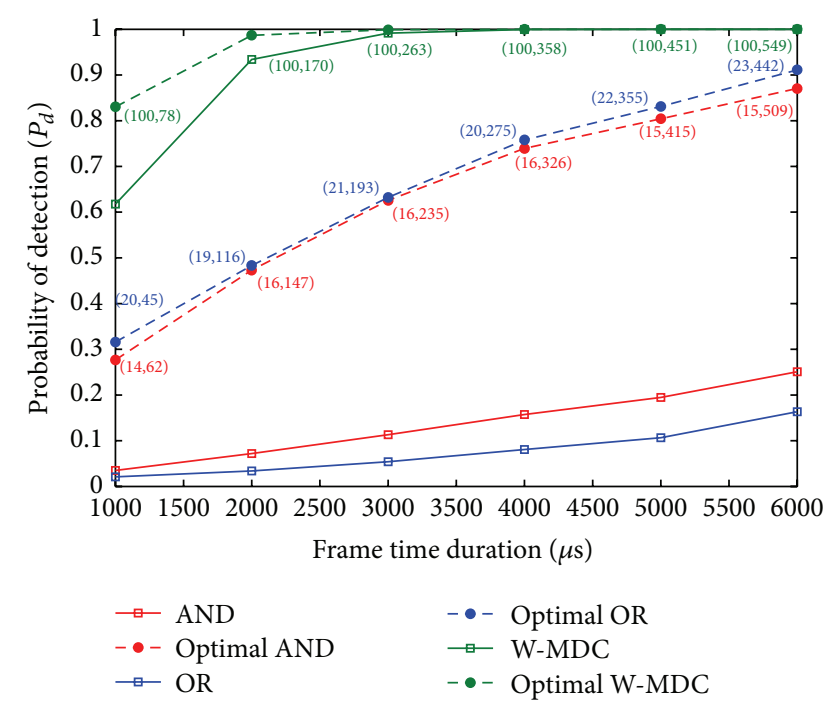

FIgURE 7: Network $P_{d}$ versus time frame duration ( $T$ in $\mu \mathrm{s}$ ) with target throughput of 0.9 for AND, OR, and weighted MDC fusion scheme; network with $100 \mathrm{SUs}$ with $\left(k_{\mathrm{opt}}, \tau_{\mathrm{opt}}\right)$ is shown in the data tips.

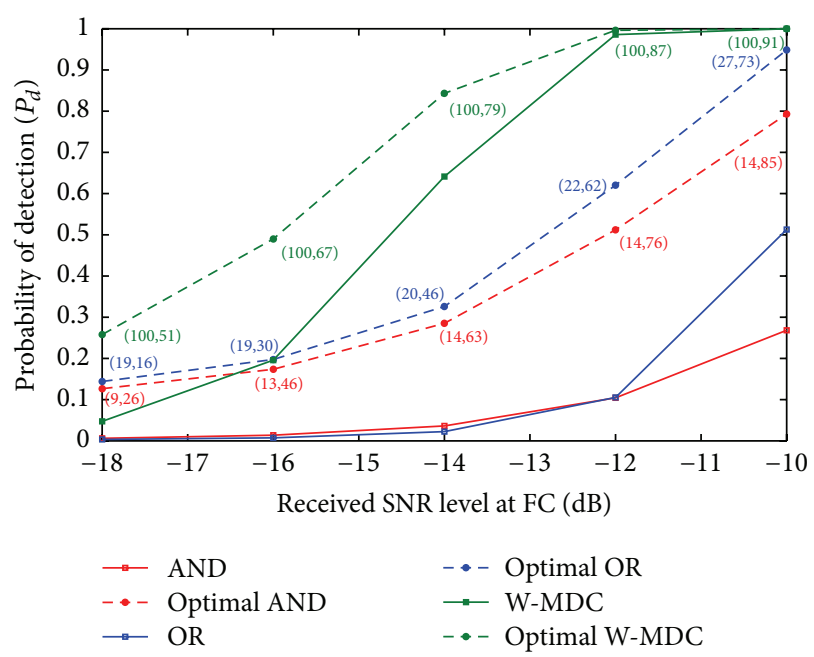

FIGURE 8: Network $P_{d}$ versus the received SNR at the fusion center (in $\mathrm{dB}$ ) for AND, OR, and weighted MDC fusion scheme; network with 100 SUs, targeted normalized throughput $=0.9$, and maximum sensing time of $\tau_{\max }=100 \mu \mathrm{s}$ with $\left(k_{\mathrm{opt}}, \tau_{\mathrm{opt}}\right)$ are shown in the data tips.

frame duration to $3000 \mu$ s and with optimizing ( $k$ and $\tau$ ), higher $P_{d}$ values are achieved and become $\approx 0.6$ for AND and OR schemes and $=0.99$ for W-MDC. Also, it can be seen that, for W-MDC fusion scheme, when the total frame duration is above $3000 \mu$ s there is almost no change in the detection performance between the optimized and nonoptimized cases.

The effect of changing the SNR levels at the fusion center on the network detection performance is investigated and presented in Figure 8. As shown, optimized W-MDC is outperforming optimized AND and OR hard fusions under the same simulation conditions in particular in high SNR 
environments. Also, as can be seen for SNR >-16 dB, nonoptimized W-MDC outperforms optimized AND and OR hard fusion schemes since the individual sensing results will improve significantly. However, in low SNR environments, optimized AND and OR can outperform the full frame sensing of $\mathrm{W}-\mathrm{MDC}$ as shown in the case of $-18 \mathrm{~dB}$.

\section{Conclusion}

In this paper, the performance of cooperative spectrum sensing is analyzed and optimized for three different fusion schemes under fixed target normalized throughput condition. The optimization is performed from SUs point of view while satisfying the PU requirement where simultaneous optimization of number of users and sensing time under novel constraint has been presented. Simulation results show that for such sensing schemes weighted MDC attains best sensing-throughput tradeoff compared to hard decision fusion. It has been shown that incorporating all secondary users in the network and using the full assigned frame for sensing time do not achieve the optimum probability of detection. Also, the paper demonstrates, with numerical examples, the exhaustive search optimization to obtain optimum values of number of cooperating users $\left(k_{\text {opt }}\right)$ that have higher primary user's signal-to-noise ratio and optimum sensing time $\left(\tau_{\text {opt }}\right)$, which is less than the maximum full frame duration under a given throughput target condition. Also, probabilities of detection with the proposed targeted normalized throughput constraint are optimized when varying network sizes, normalized target throughput, maximum frame duration times, and received signal-to-noise ratio for different fusion rules.

\section{Competing Interests}

The authors declare that they have no competing interests.

\section{Acknowledgments}

The authors would like to thank the Deanship of Scientific Research at University of Bahrain, Kingdom of Bahrain, for the financial support of this research work as part of Project no. 06-2014.

\section{References}

[1] I. F. Akyildiz, B. F. Lo, and R. Balakrishnan, "Cooperative spectrum sensing in cognitive radio networks: a survey," Physical Communication, vol. 4, no. 1, pp. 40-62, 2011.

[2] T. Yücek and H. Arslan, "A survey of spectrum sensing algorithms for cognitive radio applications," IEEE Communications Surveys \& Tutorials, vol. 11, no. 1, pp. 116-130, 2009.

[3] C. You, H. Kwon, and J. Heo, "Cooperative TV spectrum sensing in cognitive radio for Wi-Fi networks," IEEE Transactions on Consumer Electronics, vol. 57, no. 1, pp. 62-67, 2011.

[4] Y.-C. Liang, Y. Zeng, E. C. Y. Peh, and A. T. Hoang, "Sensingthroughput tradeoff for cognitive radio networks," IEEE Transactions on Wireless Communications, vol. 7, no. 4, pp. 1326-1337, 2008.
[5] E. Peh and Y.-C. Liang, "Optimization for cooperative sensing in cognitive radio networks," in Proceedings of the IEEE Wireless Communications and Networking Conference, pp. 27-32, IEEE, March 2007.

[6] Z. Quan, S. Cui, and A. H. Sayed, "Optimal linear cooperation for spectrum sensing in cognitive radio networks," IEEE Journal on Selected Topics in Signal Processing, vol. 2, no. 1, pp. 28-40, 2008.

[7] E. C. Y. Peh, Y.-C. Liang, Y. L. Guan, and Y. Zeng, “Optimization of cooperative sensing in cognitive radio networks: a sensingthroughput tradeoff view," IEEE Transactions on Vehicular Technology, vol. 58, no. 9, pp. 5294-5299, 2009.

[8] D. Sun, T. Song, M. Wu, J. Hu, J. Guo, and B. Gu, "Optimal sensing time of soft decision cooperative spectrum sensing in cognitive radio networks," in Proceedings of the IEEE Wireless Communications and Networking Conference (WCNC '13), pp. 4124-4128, IEEE, Shanghai, China, April 2013.

[9] P. Wang, L. Xiao, S. Zhou, and J. Wang, "Optimization of detection time for channel efficiency in cognitive radio systems," in Proceedings of the IEEE Wireless Communications and Networking Conference, pp. 111-115, Hong Kong, March 2007.

[10] H. Chen, L. Liu, J. Matyjas, and M. Medley, "Optimal resource allocation for sensing based spectrum sharing cognitive radio networks," in Proceedings of the IEEE Global Communications Conference, pp. 899-904, IEEE, Austin, Tex, USA, December 2014.

[11] M. Cardenas-Juarez, U. Pineda-Rico, E. Stevens-Navarro, and M. Ghogho, "Sensing-throughput optimization for cognitive radio networks under outage constraints and hard decision fusion," in Proceedings of the International Conference on Electronics, Communications and Computers (CONIELECOMP '15), pp. 80-86, IEEE, Cholula, Mexico, February 2015. 


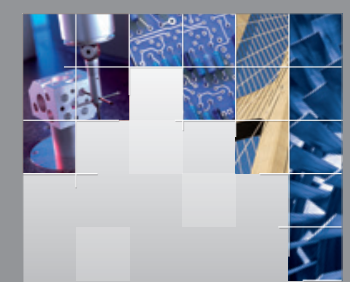

\section{Enfincering}
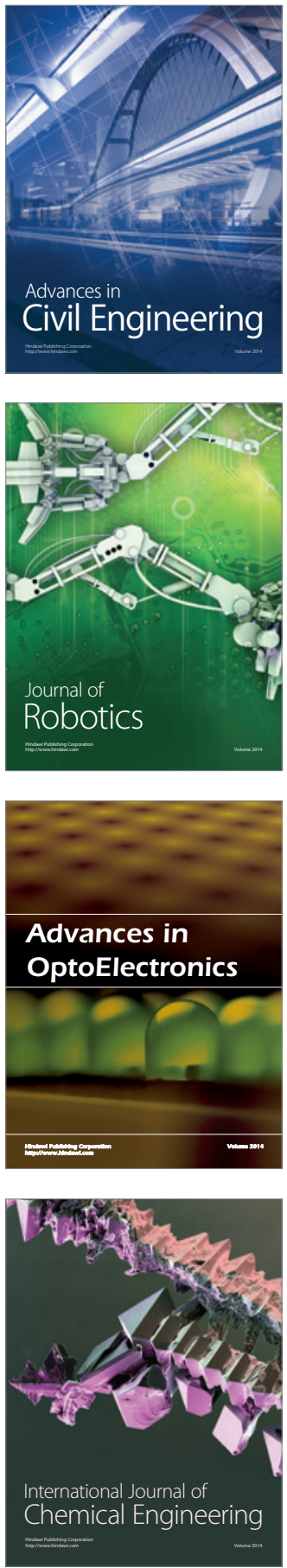

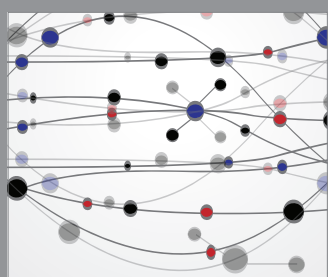

The Scientific World Journal

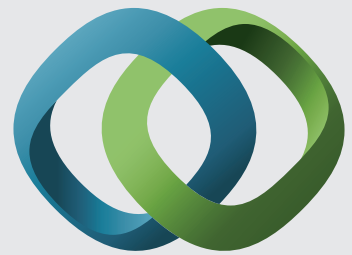

\section{Hindawi}

Submit your manuscripts at

http://www.hindawi.com
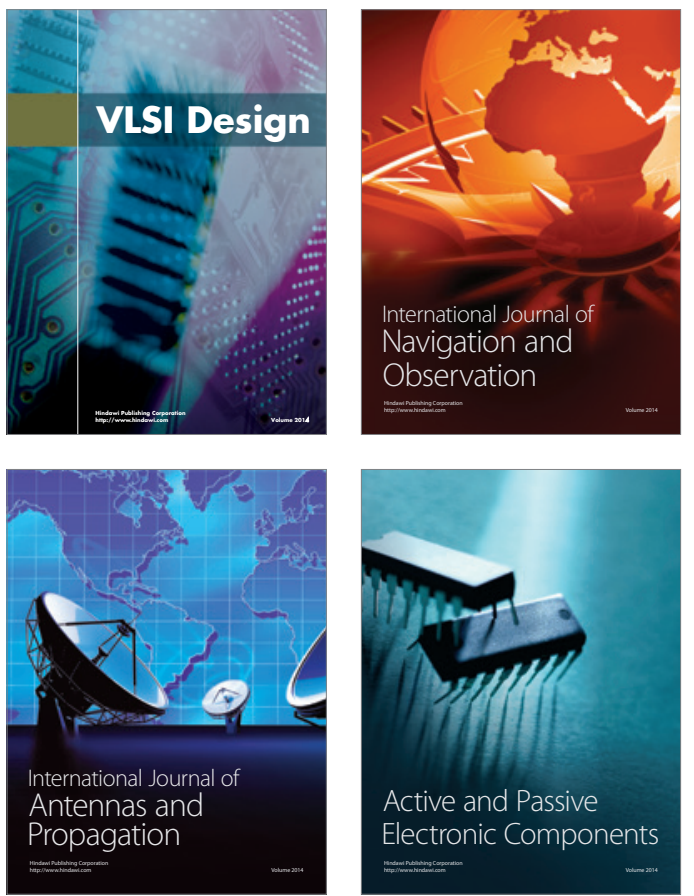
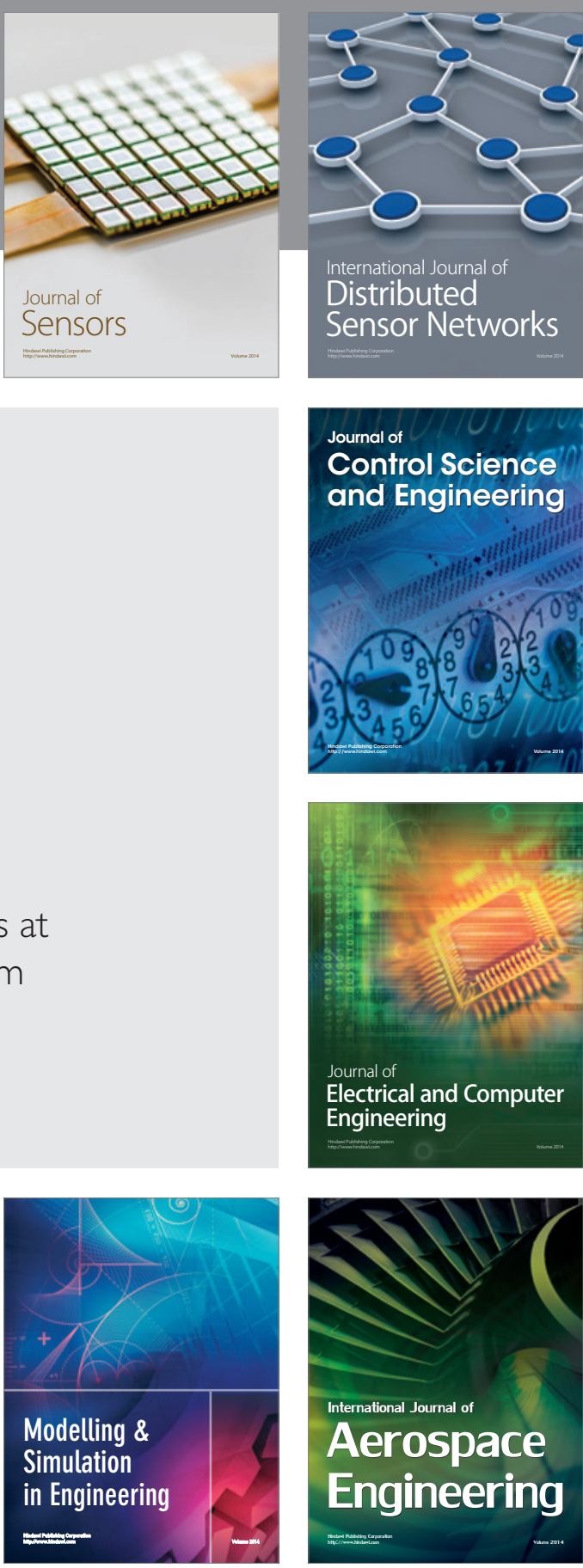

International Journal of

Distributed

Sensor Networks

Journal of

Control Science

and Engineering
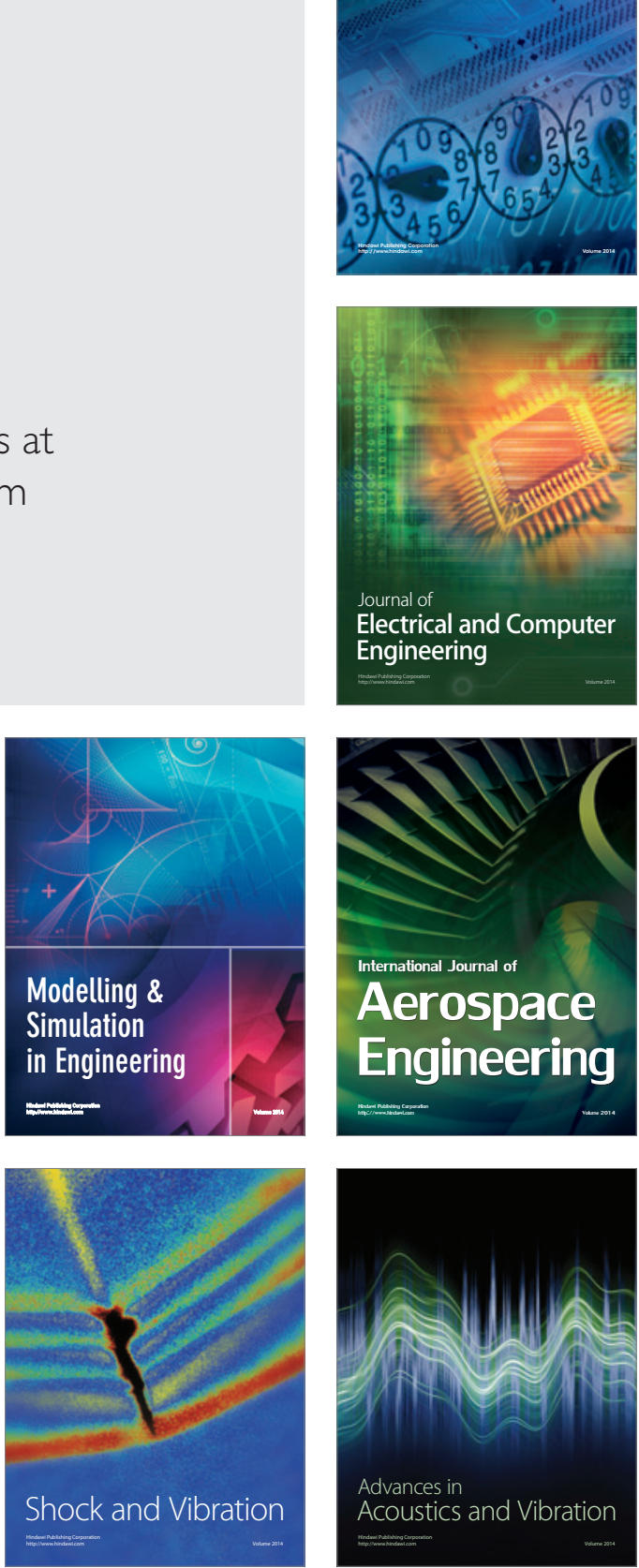